\title{
Simple and Rapid Screening Test to Detect Fake Honey
}

\section{Product}

\author{
Nor Azfa Johari ${ }^{1}$, Nur Suhanawati Ashaari ${ }^{1}$, Mohd Razif Mamat ${ }^{1}$ and Azira Muhamad ${ }^{2}$ \\ 1. Translational Research and Prototype Development Section, Malaysia Genome Institute, National Institutes of Biotechnology \\ Malaysia, Jalan Bangi, Kajang 43000, Selangor, Malaysia \\ 2. Structural Biology and Biophysics Facility Section, Malaysia Genome Institute, National Institutes of Biotechnology Malaysia, \\ Jalan Bangi, Kajang 43000, Selangor, Malaysia
}

\begin{abstract}
Despite advancement in food analytical platforms and availability of CODEX standard, the fraud honey relentlessly remains as global issue. Even with stringent guidelines, controlling the incoming honey product at ground level proved to be challenging and difficult to tackle. While most analytical platforms are powerful enough to detect counterfeit honey products, there is also a need to develop a rapid screening test to support field regulatory activity. A chemical reagent developed based on biochemistry principle has proved to be able to differentiate synthetic honey product from its raw counterpart. The reaction required only three drops of honey product to react with few drops of reagent mixtures which resulted in a change of color and precipitation of proteins and other molecules within 2 min reaction. Test performed on raw honey samples inclusive of several stingless bees and Apis species honey resulted in a precipitation as compared to fake honey generated from a blend of white sugar, golden syrup, vinegar, lime juice and plantain which resulted in clear organic phase indicating missing natural honey biological matters. The difference between raw honey and fake honey reaction with the reagents can be utilized for real time on field screening activity before proceeding with authentication using complex analytical platforms.
\end{abstract}

Key words: Raw honey, screening, fake honey, precipitation, rapid.

\section{Introduction}

Due to its high medicinal value and natural sweet sticky feature, honey has become the third most common imitated products by unscrupulous fraud producer. Regardless of the availability of international food regulation in place, the selling of fake and adulterated honey remains a major issue, which erupted as 2011 honey laundering scandal. The scandal resulted in increased awareness among public, food regulators and food analysts, as well as primed up numbers of improved complicated detection techniques. However, there is still lack of simplified or rapid technique that is able to differentiate fake product from raw honey to be applied in field investigation. For years, honey adulteration remains as

Corresponding author: Nor Azfa Johari, Ph.D., research field: biochemistry. an unresolved problem. The economically motivated activities (EMA) of honey usually involve substitution or addition of cheaper substances into raw honey to increase the volume of products and reduce the cost of production for economic gain. The EMA in honey industry includes dilution of honey with less expensive syrup, intensive supplemental feeding of bees, unapproved use of antibiotics and masking the true country of origin [1]. Honey adulteration can happen in direct and indirect way. According to Zabrodska and Vorlova [2], the direct adulteration of honey is performed by addition of adulterating substance into honey and indirect adulteration is achieved by feeding bees with adulterating substances. Other potential sources of honey contamination and adulteration might also come from the bees' contact with contaminated water, air, plants (in the case of pesticides, heavy metals, microorganism and 
genetically modified microorganism) and from the adulteration associated with processing, packaging and inadequate beekeeping practices [3]. Both methods altered the original sugar profiles of honey, where indirect adulterations remain extremely difficult to detect without sophisticated technology. Previously, The Codex Alimentarius, or "Food Code" established by the Food and Agricultural Organization (FAO) and the World Health Organization (WHO), serves as a set of voluntary international food standards, guidelines and codes of practice that contribute to the safety, quality and fairness of the international food trade [4], where, the honey quality and authenticity were studied based on Association of Official Analytical Chemists (AOAC) methods for specific parameters such as measurement of reducing sugars, ash content, electrical conductivity, 5-hydroxymethylfurfural (5-HMF) value, antioxidant activity, diastase activity and several other factors to indicate the originality of the honey. Most techniques were developed based on physicals, chemicals and biological properties of honey in singular form. Evaluations of sugars, phenolic content, total protein, amino acid, ash content, antioxidant activity, enzymes activities are among analytical methods adopted to characterize honey. However, these analytical methods are relatively time consuming, require large amount of samples, tedious preparation and various analytical equipments [5]. High-end techniques for detection of indirect adulteration have also been developed using even more complex platform such as high performance anion exchange chromatography with pulsed amperometric detection (HPAEC-PAD) [6], nuclear magnetic resonance (NMR) [7, 8], carbon-isotope ratios of different sugar sources [9] and Fourier transform infrared (FTIR) spectroscopy [10]. However, these techniques appeared to be tedious and laborious as well as not suitable for fast screening of honey product. Furthermore, due to the complex composition of honey, it is discriminative to separate individual characteristic with specific parameters, without comprehending factors such as diversity of bee species, geographical factors, climate, seasons [11], and recently new extra-floral source of nectar. The current standard for honey, Codex Standard 12-1981 has another vulnerability, where its development was based on honey bees and does not apply to stingless bees honey due to significant difference of physicochemical characteristic [12]. Honey characteristic can also change due to diverse methods in harvesting, storage conditions and processing. Harvesting method such as squash technique can affect the honey quality and may interfere with physical and chemical analysis, due to micro-leaching of propolis, which can give rise to antioxidant activity caused by excessive resin in the honey. This is due to the fact that propolis has been extensively used in folk medicine for many years, due to its antiseptic, antifungal, antibacterial, antiviral, anti-inflammatory and antioxidant properties [13]. Processing methods such as dehumidification and heating, will result in increased value of reducing sugars, sucrose and HMF as well as reduced diastase activity [14]. Straining honey from physically large matters is usually carried out to clean the product and improve the physical appearance [15], but, excessive filtering, which is unnecessary, has caused difficulties in tracing of origin based on melissopalynology or pollen identification [8]. Despite strict guidelines implemented, the mislabeling of raw and unfiltered honey has been reported by Food Safety News in a 2011 observation involving 60 honey jars containing honey without any trace of pollen [16]. The importance of carrying out routine analyses testing for authentication of honey samples has also been raised up in other country such as Saudi, since some of the investigated samples in Saudi market failed to meet the specified criteria and are thus considered not safe for human use [17]. Therefore, there is a necessity to come out with rather simple technique that is able to at least differentiate between "clear honey" and "raw honey" as the first screening step prior to further 
complicated analytical platform. Responding to this issue, a new rapid screening method has been developed to react immediately with biological matters in honey (mostly pollen and microbes) to form a solid precipitate and specific colors corresponding to the final product $\mathrm{pH}$. The developed reagents are useful for preliminary screening of marketed honey, where sugar syrup or fake honey can be differentiated from raw honey product within 2 min reaction. The technique is simple, requiring small amount of sample, and suitable for field test by regulatory bodies.

\section{Materials and Methods}

\subsection{Honey Samples and Fake Control}

Raw stingless bee honey, Apis dorsata and A. cerana honey were collected from several locations in Peninsular Malaysia. Samples were kept in a sterile amber bottle and maintained at temperature below $30{ }^{\circ} \mathrm{C}$ until test. The fake honey controls were developed from blend of white sugar and lime juice (fake control 1, FC1), blend of golden syrup, vinegar, dried tea leaves and plantain (fake control 2, FC2), honey flavour (fake control 3, FC3) and, blend of maple syrup, vinegar, dried tea leaves and plantain (fake control 4, FC4).

\subsection{The Reagents}

There were two types of reagents used in this method. The first reagent is a normal saline $(0.9 \%$ $\mathrm{NaCl}, \mathrm{pH}$ 7.0) which was used to rehydrate the honey samples. The RapidRAW reagent is developed using mixtures of organic solvents and $\mathrm{pH}$ indicator at specified ratio. Both reagents were kept in a tight high density polyethylene (HDPE) dropper bottle at temperature between $20^{\circ} \mathrm{C}$ and $30^{\circ} \mathrm{C}$ (Fig. 1).

\subsection{The RapidRAW Reaction}

A total of five drops reagent 1 is mixed with seven drops of RapidRAW reagent in a clean $1.5 \mathrm{~mL}$ micro-tube. A total of three drops of honey or fake control sample was added to the reagents and mixed vigorously until dissolved. The mixtures were left at vertical position for $2 \mathrm{~min}$ to allow for precipitation to occur. In a case of thick or dried honey, the reaction may take up to 5 min to finish. The $\mathrm{pH}$ measurement and image were recorded.

\subsection{Gas Chromatography-Mass Spectrometry (GC-MS) Profiling of Honey}

A total of $1 \mathrm{~g}$ samples were weighed in clean $2 \mathrm{~mL}$ micro-tube and diluted with $1 \mathrm{~mL}$ methanol. Phase separation was induced with $0.5 \mathrm{~mL}$ dichloromethane and samples were centrifuged at 8,000 rpm for $1 \mathrm{~min}$. The organic phase was isolated and secured for GC-MS analysis.

\section{Results}

\subsection{The Raw Honey Reactions}

3.1.1 Reactions of Various Species Stingless Bee Honey Collected from Different Locations

All raw stingless bee honey samples resulted in a precipitation and yellowish green to dark green supernatant with $\mathrm{pH}$ range from 3.00 to 5.00. Fig. 2a showed reaction profiles of honey collected from seven Indo-Malaya stingless bee species. The honey samples have a wide range of taste from sweet sour, sour to bitter sweet with pungent smell of organic acid. The reaction between RapidRAW reagent and raw honey occurred immediately upon mixing. The first reaction causes the sample color to change from yellowish brown to green. Within $2 \mathrm{~min}$, the second reaction with organic materials naturally found in raw honey causes the sample to precipitate. Gel electrophoresis of precipitates showed various sizes of protein detected with size ranging from $25 \mathrm{kDa}$ to 80 $\mathrm{kDa}$. A similar pattern can be observed from $58 \mathrm{kDa}$ to $80 \mathrm{kDa}, 30 \mathrm{kDa}$ to $46 \mathrm{kDa}$ and $25 \mathrm{kDa}$ (Fig. 2b).

3.1.2 Reactions of Apis sp. Honey and Synthetic Control

Apis honey samples of different origins showed similar reaction with RapidRAW reagent. However, 


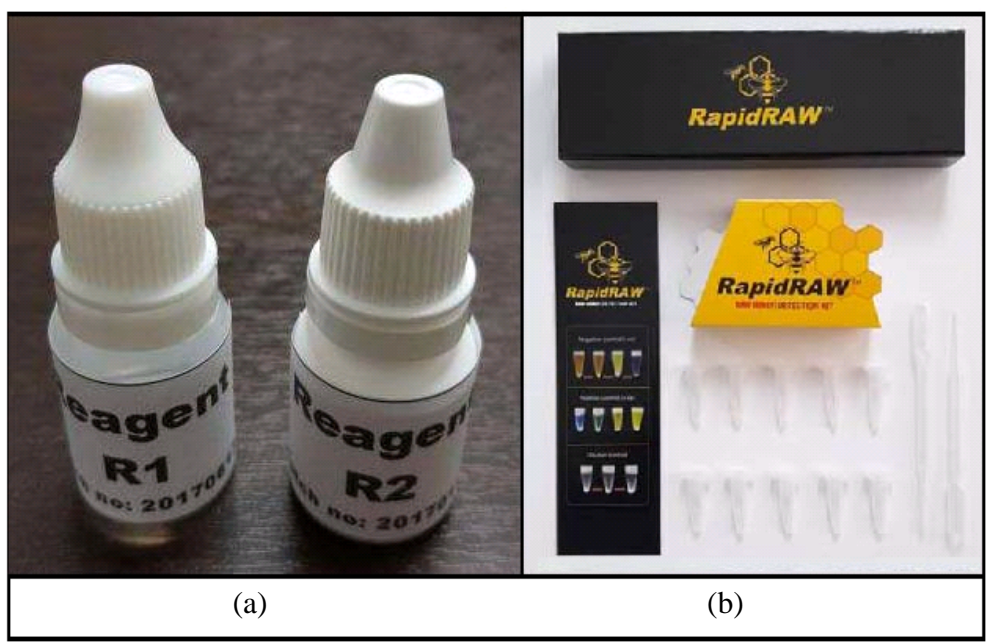

Fig. 1 The reagents stored in high density polyethylene (HDPE) dropper bottle for easy use, where: (a) normal saline pH 7.0 (Reagent R1) and RapidRAW reagent (Reagent R2); (b) RapidRAW prototype.

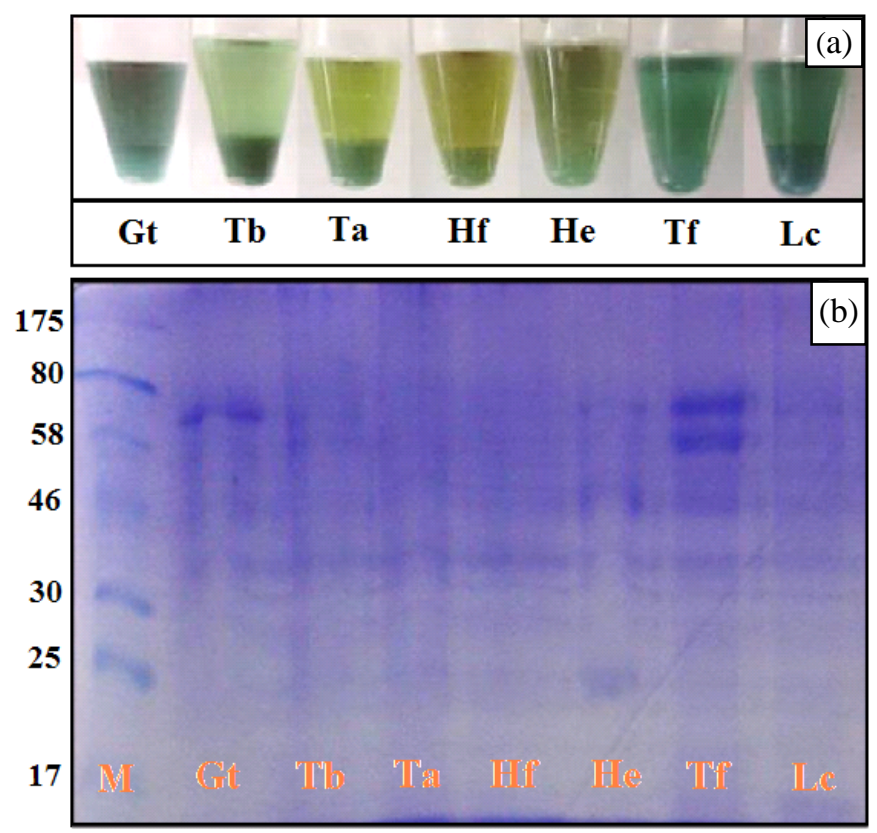

Fig. 2 RapidRAW reactions of stingless bee honey (from left: Geniotrigona thoracica (Gt), Tetrigona binghami (Tb), Tetrigona apicalis (Ta), Homotrigona fimbriata (Hf), Heterotrigona erythrogastra (He), Tetragonula fuscobalteata (Tf), Lophotrigona canifrons (Lc)) (a); Protein profiles of precipitates resulted from RapidRAW reactions of stingless bee honey (from left: Protein marker (M), Gt,Tb,Ta, Hf, He, Tf, Lc) (b).

color range of the supernatant is slightly different from stingless bee honey. Apis honey reaction resulted in green, dark green to greenish blue liquid with similar rate of precipitation and $\mathrm{pH}$ range from 3.5 to 5.5 (Fig. 3a). The protein profiles showed a protein spectrum range between $46 \mathrm{kDa}$ and $80 \mathrm{kDa}$ in all Apis honey samples (Fig. 3b). Reaction between RapidRAW reagent and fake controls resulted in color changes without precipitation, which is indicated by clear lower phase (Fig. 4). The color spectrum in fake controls is correlated with saccharine and organic acid content, where yellow and brown spectrums are indicative to high disaccharide content, and green to blue are indicative to high monosaccharide or reducing sugar content.

The same reagents somehow failed to produce any 


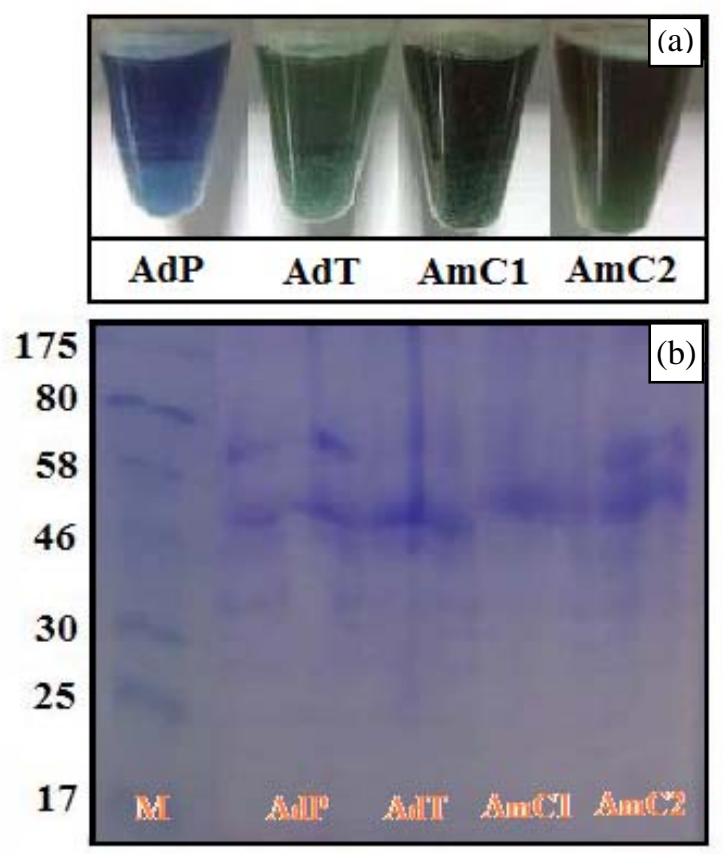

Fig. 3 RapidRAW reactions of Apis sp. honey (from left: A. dorsata Perak (AdP), A. dorsata Terengganu (AdT), A. mellifera commercial 1 (AmC1), A. mellifera commercial 2 (AmC2)) (a); Protein profiles of precipitates resulted from RapidRAW reactions of Apis sp. honey (from left: M, AdP, AdT, AmC1, AmC2) (b).

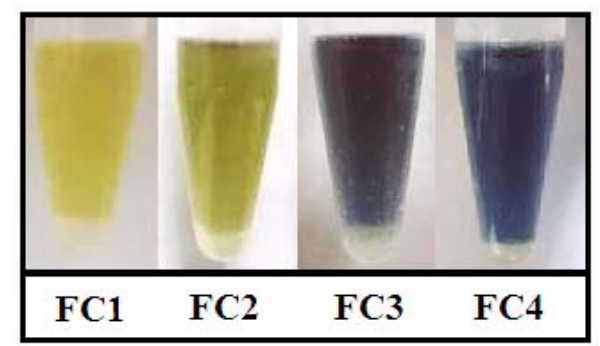

Fig. 4 RapidRAW reactions of synthetic controls.

precipitate when tested on the fake controls even with prolonged observation (up to $24 \mathrm{~h}$ after reaction) (Fig. 4). Fig. 4 showed the reaction profiles of fake control with clear lower phase and yellow supernatant (FC1) which related to high disaccharide content originated from sugarcane, green (FC2) and blue supernatants (FC3 and FC4) which related to high reducing sugar content with $\mathrm{pH}$ range from 2.00 to 6.00 .

\subsection{GC-MS Profiles of Raw and Synthetic Honey}

The organic fraction of rapid reactions subjected to GC-MS analysis showed a difference in compound profiles between raw honey and fake product (Fig. 5).
The fake control (FC1) chromatogram resulted in 5-HMF peak at retention time $8.667 \mathrm{~min}$ and low contents of resin residue. Raw honey from two different locations showed various contents of plant hydrocarbon residues at retention time 10.00-24.00 min and little to no trace of 5-HMF. A list of metabolites detected is summarized in Table 1.

\section{Discussion}

A rapid screening technique to differentiate between fake and raw honey has been developed based on the complex nature of honey. Previously, many studies focused on the individual physicochemical characteristic of honey which measure 


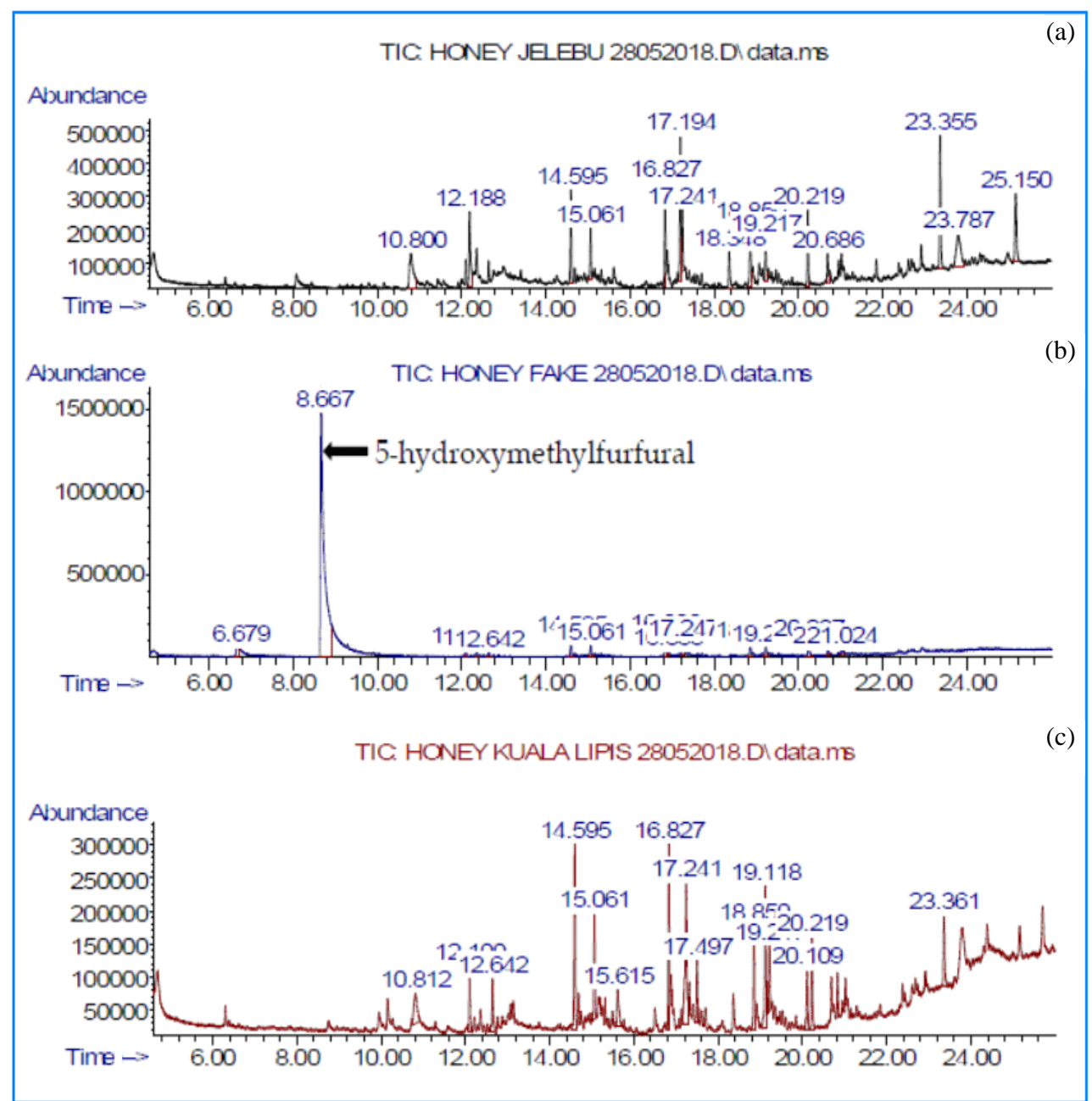

Fig. 5 Gas chromatography-mass spectrometry (GC-MS) profiles of organic fractions from raw and synthetic honeys: (a) Raw honey 1; (b) Fake honey, FC1; (c) Raw honey 2 (intensity, y-axis; minute, x-axis).

several chemical and physical parameters as indicator for authentic product. This technique however, is focusing on early screening of fake honey products, based on different carbohydrate criteria and other missing organic contents, which usually claimed as had been filtered out. The common reaction for natural honey usually results in light green to greenish blue supernatant, which is a result of sugar in acidic solution with dye used as $\mathrm{pH}$ indicator. The different supernatant color spectrum between fake control and natural honey is believed to result from the different saccharine constituent, where in this case, the fake control was developed from sucrose (FC1) and high glucose syrup (FC3 and FC4), which is a disaccharide and monosaccharide. The oxidation reaction is suspected to occur between RapidRAW reagent with hemiacetal in FC3 and FC4 to produce a dark blue supernatant. The oxidation reaction however, did not occur in FC1, probably due to missing hemiacetal structure with strong influence of synthetic acetic acid and remained as yellow supernatant. A green supernatant observed in majority of stingless bee honey reactions showed that there are complex reactions occurring between different types of sugars other than glucose and fructose, as well as significant amount of organic acids with weak reducing activity which contribute to the formation of the green spectrum.

The precipitation of protein and other insoluble matters by RapidRAW reagent is a result of organic 
Table 1 List of metabolites detected using GC-MS platform.

\begin{tabular}{|c|c|c|c|c|c|c|c|c|}
\hline \multicolumn{3}{|c|}{ Raw honey 1} & \multicolumn{3}{|c|}{ Raw honey 2} & \multicolumn{3}{|c|}{ Fake control } \\
\hline $\mathrm{RT}$ (min) & Area $(\%)$ & Library/ID & $\mathrm{RT}$ (min) & Area (\%) & Library/ID & $\mathrm{RT}$ (min) & Area $(\%)$ & Library/ID \\
\hline 10.812 & 3.7544 & Linalyl oxide & 10.8003 & 5.1211 & Trans-linalool oxide (furanoid) & 6.6851 & 3.8098 & Furyl hydroxymethyl ketone \\
\hline 12.1058 & 2.0483 & 1-iodo-Dotriacontane & 12.1934 & 4.7457 & 6-Camphenone & 8.6726 & 82.3609 & 5-hydroxymethylfurfural \\
\hline 12.6479 & 2.2358 & 1-iodo-Octadecane & 12.3566 & 2.6644 & 2,4-Di-tert-butylphenol & 16.891 & 1.0451 & $\begin{array}{l}\text { 7,9-Di-tert-butyl-1-oxaspiro(4,5 } \\
\text { )deca-6,9-diene-2,8-dione }\end{array}$ \\
\hline 14.6005 & 6.2951 & Heptacosane & 14.5947 & 4.1125 & Heneicosane & 17.2466 & 1.1374 & Octacosane \\
\hline 15.0668 & 4.0399 & $\begin{array}{l}\text { Octacosane } \\
\text { (S F) }\end{array}$ & 15.0669 & 2.5697 & 3-Ethyl-3-methylheptane & 18.8553 & 1.2457 & Octacosane \\
\hline 15.6147 & 2.1026 & $\begin{array}{l}\text { (S,E)-4-Hydroxy-3,5,5-trimethyl- } \\
\text { 4-(3-oxobut-1-en-1-yl)cyclohex-2 } \\
\text {-enone }\end{array}$ & 16.8329 & 4.5113 & Heneicosane & & & \\
\hline 16.8329 & 5.6963 & Heneicosane & 16.8854 & 2.8152 & $\begin{array}{l}\text { 7,9-Di-tert-butyl-1-oxaspiro(4,5) } \\
\text { deca-6,9-diene-2,8-dione }\end{array}$ & & & \\
\hline 16.8853 & 2.5903 & $\begin{array}{l}\text { 7,9-Di-tert-butyl-1-oxaspiro(4,5)d } \\
\text { eca-6,9-diene-2,8-dione }\end{array}$ & 17.1943 & 7.5656 & $n$-Hexadecanoic acid & & & \\
\hline 17.2409 & 7.0306 & Hentriacontane & 17.241 & 4.5507 & Heptacosane & & & \\
\hline 17.3166 & 2.2529 & Hentriacontane & 18.8497 & 3.4026 & Heneicosane & & & \\
\hline 17.4973 & 2.6261 & Hexadecanoic acid, ethyl ester & 19.2169 & 2.5893 & 1-iodo-Eicosane & & & \\
\hline 18.8496 & 3.5034 & Heneicosane & 20.2194 & 3.4714 & $\begin{array}{l}\text { Trichloroacetic acid, hexadecyl } \\
\text { ester }\end{array}$ & & & \\
\hline 19.1235 & 5.7541 & $\begin{array}{l}\text { (E)-9-Octadecenoic acid ethyl } \\
\text { ester }\end{array}$ & 23.361 & 6.9159 & Heptacosane & & & \\
\hline 19.2168 & 3.1842 & Heptacosane & 25.1504 & 5.2179 & Eicosane & & & \\
\hline 20.1086 & 2.1626 & cis-Vaccenic acid & & & & & & \\
\hline 20.2251 & 3.0782 & Octadecyl vinyl ether & & & & & & \\
\hline 23.3609 & 2.833 & Heptacosane & & & & & & \\
\hline
\end{tabular}


membrane disequilibrium caused by chemical disruption induced with solvent and mechanical force. The reagent is designed based on previous studies about solvents effect on enzyme and cell structure where dehydration caused by solvents was reported as the main process contributing to cell disruption [18, 19]. However, the reagent capacity is currently unable to differentiate $100 \%$ pure honey with low percentage of adulterated honey or contaminated pure honey. Therefore, further development is currently being carried out to improve detection of adulterated honey.

\section{Conclusions}

A new rapid screening technique to detect fake honey has been developed based on biochemistry principle of reaction between organic materials with solvents and halogen group at specific designated ratio. The duration for complete precipitation of organic materials in raw honey depends on the initial content of intact organic materials in sample, while the supernatant color after precipitation depends on the saccharine composition. This technique when applied to raw and fake honey resulted in a distinct pattern of reaction, thus making it possible to differentiate between the two products. The outcome is a simple and rapid screening of honey product which allows for real-time detection of fake honey using minimum amount of sample. Further investigation is currently being carried out to characterize the reaction according to percentage of adulteration to determine the sensitivity of this technique. At the same time, the reagent differentiation capacity is currently being verified using NMR platform, for non-biased random profile analysis.

\section{Acknowledgments}

Special thanks go to Mr. Abu Hassan Jalil from Akademi Kelulut Malaysia for sharing his expertise in bees and honey industry. Authors would also like to thank other collaborators, Great Farm, The Bees Alchemy, Madu Kelulut Merbuk SAF, Madu Kelulut
Raw Trigona Honey, Syamille Agrofarm and Trigona Asli for their sample contribution as test material.

\section{References}

[1] Strayer, S. E., Everstine, K., and Kennedy, S. 2014. "Economically Motivated Adulteration of Honey: Quality Control Vulnerabilities in the International Honey Market.” Food Protection Trends 34 (1): 8-14.

[2] Zabrodska, B., and Vorlova, L. 2015. "Adulteration of Honey and Available Methods for Detection-A Review.” Acta Vet. Brno 83 (10): S85-102.

[3] Soares, S., Amaral, J. S., Oliveira, M. B., and Mafra, I. 2017. "A Comprehensive Review on the Main Honey Authentication Issues: Production and Origin." Comprehensive Reviews in Food Science and Food Safety 16: 1072-100.

[4] Codex Alimentarius Commission. 2013. Codex Alimentarius International Food Standards. Accessed September 6, 2019. http://www.fao.org/fao-who-codexalimentarius/en/.

[5] Cozzolino, D., Corbella, E., and Smyth, H. E. 2011. "Quality Control of Honey Using Infrared Spectroscopy: A Review.” Applied Spectroscopy Reviews 46: 523-38.

[6] Cordella, C. H., Militao, J. S. L. T., Clement, M. C., Drajnudel, P., and Cabrol-Bass, D. 2005. "Detection and Quantification of Honey Adulteration via Direct Incorporation of Sugar Syrups or Bee-Feeding: Preliminary Study Using High Performance Anion Exchange Chromatography with Pulsed Amperometric Detection (HPAEC-PAD) and Chemometrics.” Analytica Chimica Acta 531: 239-48.

[7] Bertelli, D., Lolli, M., Papotti, G., Bortolotti, L., Serra, G., and Plessi, M. 2010. "Detection of Honey Adulteration by Sugar Syrups Using One-Dimensional and Two-Dimensional High-Resolution Nuclear Magnetic Resonance.” Journal of Agricultural and Food Chemistry 58: 8495-501.

[8] Ohmenhaeuser, M., Monakhova, Y. B., Kuballa, T., and Lachenmeier, W. 2013. "Qualitative and Quantitative Control of Honeys Using NMR Spectroscopy and Chemometrics.” ISRN Analytical Chemistry, Article ID 825318. http://dx.doi..org/10.1155/2013/825318.

[9] Guler, A., Kocaokutgen, H., Garipoglu, A. V., Onder, H., Ekinci, D., and Biyik, S. 2014. "Detection of Adulterated Honey Produced by Honeybee (Apis mellifera L.) Colonies Fed with Different Levels of Commercial Industrial Sugar (C3 and C4 Plants) Syrups by the Carbon Isotope Ratio Analysis.” Food Chemistry 155: 155-60.

[10] Wang, J., Kliks, M. M., Jun, S., Jackson, M., and Li, Q. X. 2010. "Rapid Analysis of Glucose, Fructose, Sucrose and Maltose in Honeys from Different Geographic Regions Using Fourier Transform Infrared Spectroscopy and 
Multivariate Analysis.” Journal of Food Science 75: 208-14.

[11] Carvalho, C. A. L., Sodre, G. S., Fonseca, A. A. O., Alves, R. M. O., Souza, B. A., and Clarton, L. 2009. "Physicochemical Characteristics and Sensory Profile of Honey Samples from Stingless Bee (Apidae: Meliponinae) Submitted to a Dehumidification Process." Anais da Academia Brasileira de Ciencias 81 (1): 143-9.

[12] Vit, P. 2008. "The Pre-columbian Honey Bees (Meliponini) Has Not Yet Quality Standards.” Bulletin Biological Research Centre 42 (3): 415-23.

[13] Galeotti, F., Maccari, F., Fachini, A., and Volpi, N. 2018. "Chemical Composition and Antioxidant Activity of Propolis Prepared in Different Forms and in Different Solvents Useful for Finished Products.” Foods 7 (3): 41. doi: $10.3390 /$ foods7030041.

[14] White Junior, J. W. 1994. "The Role of HMF and Diastase Assays in Honey Quality Evaluation.” Bee World 75 (3): 104-17.

[15] Bryant, V. 2017. "Filtering Honey Almost Every Filter Removes Some Pollen.” Accessed September 6, 2019. https://www.beeculture.com/filtering-honey-almost-every -filter-removes-pollen/.
[16] Schneider, A. 2011. “Test Show Most Store Honey Isn’t Honey.” Accessed September 6, 2019. https://www.foodsafetynews.com/2011/11/tests-show-mo st-store-honey-isnt-honey/.

[17] Aljohar, H. I., Maher, H. M., Albaqami, J., Al-Maheizie, M., Orfali, R., Orfali, R., and Alrubia, S. 2018. "Physical and Chemical Screening of Honey Samples Available in the Saudi Market: An Important Aspect in the Authentication Process and Quality Assessment.” Saudi Pharmaceutical Journal 7 (26): 932-42

[18] Yang, M., Wu, H., Lian, Y., Lai, F., and Zhao, G. 2014. "Influence of Organic Solvents on Catalytic Behaviours and Cell Morphology of Whole-Cell Biocatalyst for Synthesis of 5'-Arabinocytosine Laurate." PLOS One 9 (8): e104847. doi: 10.1371/journal.pone.0104847.

[19] Baumgarten, T., Vazquez, J., Bastisch, C., Veron, W., Feuilloley, M. G., Nietzsche, S., Wicky, L. Y., and Heipieper, H. J. 2012. “Alkanols and Chlorophenols Cause Different Physiological Adaptive Responses on the Level of Cell Surface Properties and Membrane Vesicle Formation in Pseudomonas putida DOT-T1E.” Applied Microbiology and Biotechnology 93 (2): 837-45. 